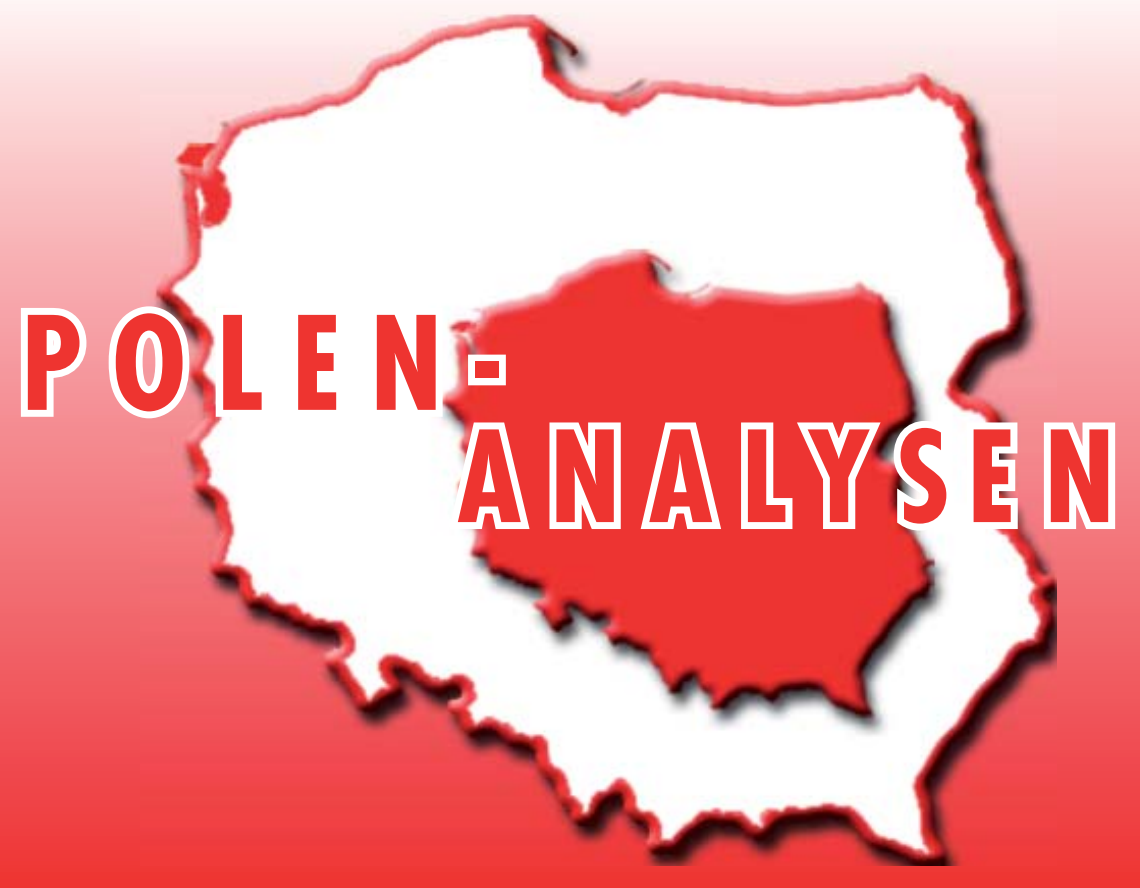

www.laender-analysen.de/polen

\title{
POLNISCHE PRESSE
}

ANALYSE

Die Presselandschaft in Polen. Strukturelle Rahmenbedingungen und zentrale Konfliktlinien

Johanna Möller, Bremen

- TABELLEN UND GRAFIKEN

Auflage und Verkauf polnischer Printmedien

CHRONIK

Vom 07. bis zum 20. April 2009 


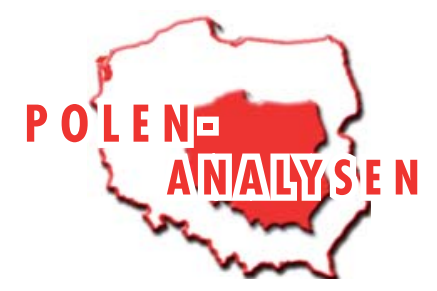

Analyse

\section{Die Presselandschaft in Polen. Strukturelle Rahmenbedingungen und zentrale Konfliktlinien}

Johanna Möller, Bremen

\section{Zusammenfassung}

Seit dem Zusammenbruch des kommunistischen Systems hat sich die polnische Presselandschaft außerordentlich dynamisch entwickelt. Trotz konzentrierter Besitzstrukturen im Bereich der politischen Tages- und Wochenpresse - diese befindet sich zu einem großen Teil in den Händen deutscher Medienkonzerne - hat sich ein vielfältiges Medienangebot etabliert. Auch die politisch-rechtlichen Rahmenbedingungen für journalistisches Arbeiten sind im Großen und Ganzen als positiv zu beurteilen - der Zugang zu Informationen ist ebenso gewährleistet wie der Schutz der Meinungsvielfalt. Probleme wie zurückgehende Leserzahlen, Boulevardisierung der Medieninhalte und zunehmender finanzieller Druck auf Journalisten sind auch für westeuropäische Medien gut bekannt. Darüber hinaus sind für die polnische Presselandschaft spezifische Konflikte prägend, die um ideologische Lagerbildung und politische Einflussnahme seitens der Journalisten kreisen und das Vertrauen der Gesellschaft in die Presse als kritischer Politikbegleiter immer wieder erschüttern.

$\mathrm{V}$ on 1989 bis heute hat sich der polnische Pressemarkt außerordentlich dynamisch entwickelt. Blickt man auf die letzten 20 Jahre zurück, so zeigt sich, dass die aktuelle Situation kaum mehr aus der Perspektive einer politischen Transformation beschrieben werden kann. Die polnische Presselandschaft verfügt über ein vielfältiges Angebot politischer Tages- und Wochenzeitungen. Freie Meinungsäußerung und Meinungsvielfalt sind ebenso gewährleistet wie ein breites Spektrum unterschiedlichster Standpunkte. Die politische Tages- und Wochenpresse in Polen kämpft nicht mit staatlichen Zensurbehörden, sondern vielmehr mit sinkenden Leserzahlen und finanziellem Druck seitens der Verleger. Diese strukturelle Problematik ist auch für die westeuropäische Presse mehr als bekannt.

Das demokratische Polen startete mit hohen Idealen und einem ebenso hohen Anspruch an die Presse. Die für die Presse zentrale Entscheidung am »Runden Tisch" war die Auflösung des Monopols des staatlichen Herausgebers Robotnicza Spółdzielnia Wydawnicza "Prasa Książka Ruch" (RSW). Jeder volljährige und nicht vorbestrafte Bürger konnte nun einen Pressetitel gründen oder erwerben. Dieser Schritt bewirkte, dass neue Titel massenhaft entstanden und alte Medien übernommen wurden - auch durch ausländische Konzerne, die freien Zugang zum Pressemarkt erhielten und von dieser Möglichkeit intensiv Gebrauch machten. Nach einer turbulenten Phase, in der der Markt ständigen Änderungen unterlag, kam es ab 1996 zu einer vorläufigen Beruhigung und Ordnung des Marktes, auf dem sich seit Beginn dieses Jahrzehnts schließlich nach und nach die gegenwärtige Struktur etablierte.
Dieser Artikel setzt sich zunächst mit den strukturellen Bedingungen der polnischen politischen Presselandschaft auseinander, um anschließend entscheidende Akteurskonstellationen und Konfliktbereiche und damit die inhaltliche Ebene in den Blick zu nehmen. Gerade diese Perspektive verdeutlicht die Spezifika der polnischen Presselandschaft. Im Mittelpunkt der nachfolgenden Überlegungen steht die sogenannte meinungsbildende Presse, die die politisch-kommentierenden Printmedien (Tages- und Wochenzeitungen) umfasst. Querverweise auf den Rundfunk und andere Medien dienen der Kontextualisierung.

\section{Medienmarkt und -angebot}

Das Angebot des polnischen Pressemarkts ist pluralistisch, bunt und umfangreich und hat in den vergangenen Jahren insgesamt stetig zugenommen. Diese Entwicklung gilt besonders für den Zeitschriftenmarkt. Den Bereich der Tagespresse bestimmten lange Gazeta Wyborcza und Rzeczpospolita. Mit der linksliberalen Gazeta Wyborcza, die ihre Wurzeln in der Oppositionsbewegung Solidarność hat, auf der einen und der konservativen Zeitung Rzeczpospolita, deren Geschichte noch in die Volksrepublik zurückreicht, auf der anderen Seite war das Angebot an polnischen Qualitätszeitungen bereits beschrieben. Erst 2006 kam als Konkurrenz der liberal-konservative Dziennik aus dem Hause Springer dazu. Die Existenz dieser mittelstarken Zeitung stand zwischenzeitlich immer wieder zur Debatte, sie konnte sich jedoch bisher am Markt halten - eine Entwicklung, die positiv zu beurteilen ist, erweitert sich damit doch das Spektrum der Qualitätspresse um ein Blatt der kon- 


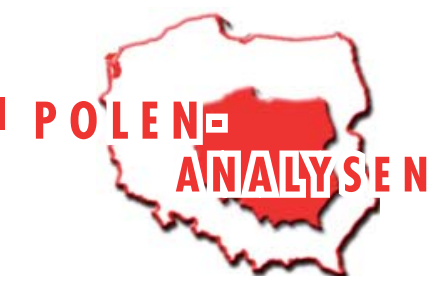

polen-analysen 50/09

servativen Mitte. Die Gazeta Wyborcza wie auch die Rzeczpospolita hatten sich tendenziell eher mit polarisierenden Positionen am Markt behauptet.

Bei den Boulevardzeitungen dominiert das SpringerBlatt Fakt, das bereits direkt nach seinem Markteintritt die Führungsposition bei den Tageszeitungen einnehmen konnte und damit nicht nur die Gazeta Wyborcza als auflagenstärkste Zeitung ablöste, sondern auch seinen Konkurrenten Super Express auf die hinteren Ränge verwies. Nachdem einer der Fakt-Vizechefredakteure als Chefredakteur zu Super Express gewechselt hat, sind die beiden Blätter in Form und Inhalt kaum noch voneinander zu unterscheiden. Dennoch konnte Super Express nicht zur Auflagenstärke von Fakt aufschließen. Deren Format orientiert sich stark an ihrem Vorbild, der deutschen Bild-Zeitung, zeigt aber inhaltlich ganz eigene Charakteristika. So entstand auf den Seiten 2 und 3 eine Meinungsseite, auf der externe Experten zu aktuellen politischen Themen Stellung beziehen. Das »Seite-1Mädchen« befindet sich auf der letzten, statt auf der ersten Seite und magische Naturheiler stellen bioenergetisch aufgeladene Heilgürtel zum Ausschneiden bereit.

Im Vergleich zu dem relativ überschaubaren Spektrum auf dem Tagespressemarkt ist das Angebot an politischen Wochenzeitungen recht beachtlich. Marktführer ist hier das Magazin Polityka, welches zusammen mit dem kleineren Magazin Przekrój im politischen MitteLinks-Bereich anzusiedeln ist. Auf der anderen Seite stehen die tendenziell konservative Newsweek Polska und das konservativ-nationalistische Magazin Wprost, das immer wieder mit provokativen Titelblättern von sich reden machte. Unter den viel gelesenen Blättern befindet sich außerdem das katholische Magazin Gość Niedzielny, welches mit fast ausschließlich religiösen Inhalten seine Auflagenzahlen zuletzt sogar steigern konnte.

Bei den Besitzerstrukturen dominieren nicht nur im Rahmen der politischen Tagespresse ausländische, zumeist deutsche Medienkonzerne. Neben dem Haus Burda, das im Bereich der Regenbogenpresse eine absolute Spitzenstellung einnimmt, ist Axel Springer mit der auflagenstarken Boulevardzeitung Fakt, dem Wochenblatt Newsweek und dem Qualitätsblatt Dziennik Marktführer der politischen Presse. Ein weiterer großer Medienanbieter ist der Verlag Polskapresse aus dem Haus Neue Passauer Presse. Dieser ebenfalls deutsche Investor ist vor allem auf dem regionalen Tagespressemarkt engagiert und in allen polnischen Regionen präsent - mit insgesamt 18 Blättern. Ein weiterer ausländischer Player ist die norwegische Gruppe Orkla Press, die in Polen unter dem Label Presspublica aktiv ist. Anders als im Bereich der elektronischen Medien, in dem der
Nationale Landesrundfunk- und Fernsehrat (Krajowa Rada Radiofonii i Telewizji, KRRiT) für die Vergabe von Lizenzen zuständig ist, ist der Zugang zum polnischen Pressemarkt vergleichsweise frei geregelt. Deutsche Konzerne nehmen so beispielsweise die Möglichkeiten zur Expansion auf dem polnischen Markt gerne in Anspruch.

Als einziger polnischer Medienkonzern zählt die Aktiengesellschaft Agora S.A. als Herausgeberin der Gazeta Wyborcza zu den großen Medienplayern auf dem polnischen Pressemarkt. Ein für die Presselandschaft ökonomisch kleiner, politisch aber zentraler Akteur ist darüber hinaus die polnische Regierung, vermittelt durch das Schatzministerium. Dieses besitzt einen 80-prozentigen Anteil an dem Verlagshaus PWR (Przedsiębiorstwo Wydawnicze »Rzeczpospolita»), das wiederum 49 Prozent der Anteile an der Tageszeitung Rzeczpospolita hält, und kontrolliert die öffentlich-rechtlichen Fernseh- und Rundfunksender sowie die größte Nachrichtenagentur Polska Agencja Prasowa (PAP).

Die starke Dominanz großer, zumeist ausländischer Medienkonzerne auf dem polnischen Pressemarkt hat immer wieder die Diskussion um drohende Monopolbildungen befeuert. Zuletzt interessierte sich der SpringerKonzern intensiv für die Anteile der polnischen Regierung an der konservativen Rzeczpospolita. Nach einer Prüfung der Bücher der angeschlagenen Tageszeitung kam jedoch der Rückzug - innerhalb der gesetzten Frist ging kein Angebot des Konzerns ein. Mit dem Erwerb dieser und möglicherweise auch anderer Anteile an der Zeitung hätte Springer die Gazeta Wyborcza noch stärker als bisher »in die Zange« genommen. Vermutlich hätte dies auch das Ende des Springer-Blatts Dziennik bedeutet und damit das Angebot nationaler Qualitätszeitungen wieder auf zwei Blätter reduziert.

Vor dem Hintergrund solcher Ereignisse ist die kritische Diskussion um Monopolbildungen auf dem polnischen Medienmarkt zumindest verständlich, allerdings nicht bis zum Schluss nachvollziehbar. Gerade die Aktivität des Springer-Konzerns auf dem Markt der politischen Tages- und Wochenpresse hat für eine verstärkte Pluralisierung des Angebots, auch inhaltlicher Art, in diesem Bereich gesorgt. Die Befürchtung, Springer würde über seine Formate »deutsche Positionen« in Polen vertreten, bestätigte sich nicht - im Gegenteil zeichnet beispielsweise die Zeitung Fakt immer wieder für deutschlandfeindliche Texte verantwortlich.

Davon abgesehen haben Tages- und Wochenzeitungen in Polen durchgehend mit zurückgehenden Auflagenzahlen zu kämpfen. Das in allen Themenbereichen (Fernsehen, Inneneinrichtung, Frauen etc.) zunehmende 


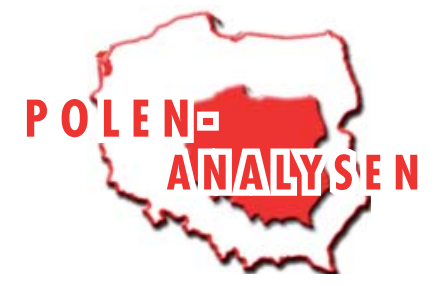

polen-analysen 50/09

Zeitschriftenangebot geht zu Lasten der bestehenden Magazine, insbesondere aber der Regionalpresse, die in den vergangenen Jahren erhebliche Verluste hinnehmen musste. Entscheidenden Einfluss hatten hier neben der bereits erwähnten, durch Fakt verursachten Zäsur u. a. die Gratiszeitungen. Diese hatten sich zunächst als Massenphänomen auf dem polnischen nationalen, später auch lokalen Pressemarkt gezeigt, letztlich überlebten jedoch nur zwei Titel - Metro, herausgegeben von Agora, und Echo Miasta, ein Blatt aus dem Hause Polskapresse mit Ausgaben in sieben polnischen Städten. Die Gratiszeitung Metropol, herausgegeben von einem schwedischen Konzern, musste 2007 die Arbeit einstellen. Die Gratisblätter, so zeigte sich, sind dann in der Lage zu überleben, wenn große Verlagshäuser hinter ihnen stehen und sie sinnvoll mit anderen Medienangeboten kombiniert werden können. So kann beispielsweise das Haus Agora Medieninhalte auch über die Gratiszeitung Metro verwerten und von den Einnahmen aus deren Anzeigen profitieren. Nicht zuletzt ist das Internet eine wachsende Konkurrenz für die politische Tagesund Wochenpresse.

Ebenso wie in anderen westlichen Ländern ist die polnische Tagespresse in zunehmendem Maße nicht nur vom Verkauf der Zeitung, sondern auch von den Einnahmen aus der Werbung abhängig. Der wachsende Werbemarkt lässt sich gut an der steigenden Anzahl spezialisierter Magazine ablesen (Frauen- und Männermagazine, Hobbys etc.), deren Publikationsstrategie zum großen Teil auf Werbung basiert. Aber auch die politische Tages- und Wochenpresse in Polen ist fast schon zu gleichen Teilen von der Werbung wie von den Verkaufszahlen abhängig. Dem wirtschaftlichen Druck, der für alle Tageszeitungen gerade auch im Rahmen der internationalen Finanzkrise zunehmend eine Belastung wird, versucht auch die Verlagsgruppe Polskapresse mit einer rationalisierten Publikationsstrategie zu begegnen. So produziert die Regionalzeitungsgruppe den gesamten politischen Mantel (nationale und internationale Politik) unter anderem unter Verwendung von Artikeln der Londoner Times zentral in Warschau und stellt ihn den lokalen Redaktionen zur Verfügung. Diese haben keinen Einfluss mehr auf die Inhalte. Neuerdings muss das Haus in dieser Frage jedoch zurückrudern und überlässt den regionalen Zeitungen wieder die Gestaltung der Titelseiten - die Verkaufszahlen waren zu stark gesunken, was mit einer schwachen regionalen Bindung an das Blatt in Verbindung gebracht wurde.

Am Beispiel der Wochenzeitung Tygodnik Powszechny (Auflage um 40.000) lässt sich illustrieren, wie sehr die aktuelle Finanzkrise die Situation auf dem Werbemarkt verschärft hat und damit eine wichtige Einkommensquelle der Printmedien bedroht. Die katholisch-liberale Wochenzeitung kann auf eine Tradition seit 1945 sowie viele bekannte Redaktionsmitglieder verweisen, die in der demokratischen Bewegung der 1970er und 1980er Jahre eine wichtige Rolle spielten. Sie hat ihre feste Klientel unter den katholischen Intellektuellen Polens. Während der Zeit des Kommunismus war das Blatt die kritischste polnische Zeitung, heute gerät es mit seiner Redaktionslinie vor allem mit konservativen Vertretern der katholischen Kirche in Konflikt. Nach einem drastischen Einbruch der Auflagenzahlen sah sich der Chefredakteur gezwungen, auf dem Titelblatt der Zeitung seine Leser um Spenden zu bitten - noch scheint sie sich damit am Leben zu halten.

\section{Politisch-rechtliche Rahmenbedingungen}

Die rechtlichen Rahmenbedingungen für das polnische Mediensystem zeigen einen eigentümlichen Widerspruch: Während der Gesetzgeber im Bereich der elektronischen Medien erst 2005 eine neue Regelung bereitgestellt hat, datiert das polnische Pressegesetz noch aus dem Jahr 1984. Die Gesetzgebung im Bereich Hörfunk und Fernsehen wurde im Laufe der Jahre immer wieder erneuert, im Bereich Presse wurden dagegen nur Anpassungen und kleinere Änderungen vorgenommen. Eine wesentliche Neuerung, gültig seit 2002, bestimmt zum Beispiel die Verpflichtung öffentlicher Institutionen, "der Presse Auskunft über ihre Tätigkeit zu erteilen", es sei denn, es gilt Geheimhaltung (Pressegesetz Art. 4, Abs. 1). Die Rechtspraxis in Polen beschränkt sich jedoch nicht auf diesen Text. Über das Pressegesetz hinaus wird der rechtliche Rahmen für die journalistische Tätigkeit in der Verfassung und in einigen anderen Gesetzen, unter anderem im Strafgesetzbuch der Republik Polen, geregelt. Über die Verfassung wird die Meinungsfreiheit sowie die Beschaffung und Verbreitung von Informationen gesichert. Der Anspruch auf Gewährleistung des Zugangs zu Informationen sowie des Schutzes von Geheimnissen und Informanten wird garantiert.

Die Gesetzgebung verbietet damit Formen direkter und indirekter Zensur. Die praktischen Beschränkungen des Presserechts, vor allem aber die Rechtspraxis, die sich auch auf andere Rechtsquellen beruft, machen die Lage jedoch weniger eindeutig. Über die Regelung im Strafgesetzbuch eröffnen sich Möglichkeiten von Missbrauch - sowohl auf der Seite der Journalisten als auch auf der Seite von Politikern oder anderen einflussreichen Akteuren in der Gesellschaft. 


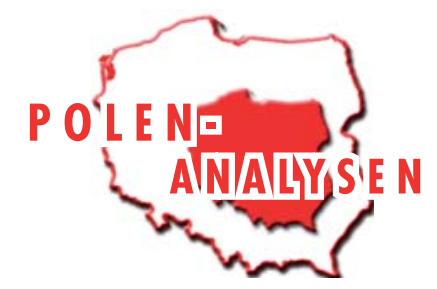

Möglichkeiten, unliebsame journalistische Positionen anzugreifen, stellen insbesondere die Artikel 212 (»Verleumdung«) und 226 (»Beleidigung einer öffentlichen Person«) StGB dar, die immer wieder als »versteckte Zensur « gegen Journalisten eingesetzt werden - und dies in jüngster Vergangenheit in zunehmendem Maße. Diese Artikel (wie auch andere) beziehen sich nicht direkt auf das Presserecht, sie werden aber immer wieder für Klagen gegen Journalisten herangezogen, die sich wiederholt gegen die Gesetzestexte zur Wehr setzen. So tritt die Vereinigung Polnischer Journalisten (Stowarzyszenie Dziennikarzy Polskich, SDP) dafür ein, die Verantwortung für Artikel nicht nur den Journalisten zu überlassen, sondern auch die Herausgeber miteinzubeziehen und eine solche Regelung in einem neuen Pressegesetz zu verankern. Eine Verurteilung nach den oben genannten Artikeln ist keine Kleinigkeit - ein Verstoß kann mit bis zu drei Jahren Gefängnis und hohen Geldstrafen geahndet werden. Erst kürzlich wurde der Fall einer Journalistin der Boulevardzeitung Super Express bekannt, die über einen angeblich pädophilen Mann berichtete. Es stellte sich heraus, dass nicht er, sondern sein Bruder sich strafbar gemacht hatte. Der Verdächtigte verklagte die Journalistin, die nun eine zweijährige Haftstrafe antreten muss.

Formen »indirekter Zensur« zeigten sich in der Phase der politischen Führung unter den Brüdern Jarosław (Ministerpräsident von Juli 2006 bis November 2007) und Lech (Staatspräsident seit 2005) Kaczyński in besonderem Maße und vermehrt auf der politischen Ebene. Die Machtübernahme von Recht und Gerechtigkeit (Prawo i Sprawiedliwość-PiS), der Partei der Kaczyńskis, war hier eine Zäsur. Dies zeigte und zeigt sich besonders krass am Fall des KRRiT. Bereits im Vorfeld seines Amtsantritts hatte Jarosław Kaczyński immer wieder angekündigt, die politische Kontrolle über die Medien erhöhen zu wollen. Dieses Vorhaben wurde ungewöhnlich rasch umgesetzt, und zwar unter anderem auch auf dem Feld der Gesetzgebung. In dem bisher schnellsten Gesetzgebungsverfahren in Polen spielte der KRRiT eine wichtige und umstrittene Rolle. Diese Institution existiert seit 1992 und hat in erster Linie die Vergabe von Sendelizenzen an Hörfunk- und Fernsehanstalten zur Aufgabe. Abgesehen davon, dass das neue Gesetz über die Kompetenzen des Rates eine Benennung des Ratsvorsitzenden durch den Staatspräsidenten festlegte, ermächtigte es ihn »zu Anregungen und Handlungen im Bereich journalistischer Ethik«. In der Folge wurden immer wieder Fälle bekannt, in denen der Rundfunkrat unethische journalistische Berichterstattung im Sinne der PiSEthik ahndete und mit Geldbußen sanktionierte. Der
Versuch der politischen Beeinflussung der Medien über den KRRiT oder auch über andere Wege sind zwar kein neues Phänomen in Polen - die Regierung Kaczyński konnte aber den Einfluss der Regierung in einem bislang nicht erreichten Umfang geltend machen.

Die vielfach geforderte Änderung der aktuellen Mediengesetzgebung bereitet zahlreiche Probleme. Neben mit großer Sicherheit zu erwartenden harten politischen Auseinandersetzungen über den Inhalt gilt es, eine solche Gesetzgebung in den europäischen und internationalen Rechtsrahmen einzupassen. Das würde bedeuten, dass ein völlig neues Projekt erstellt werden müsste. Absichten dazu werden regelmäßig bekundet, vor dem konkreten Schritt schrecken die polnischen Politiker allerdings noch zurück.

Die Regierung von Ministerpräsident Donald Tusk (seit November 2007 im Amt) hat seit ihrem Antritt immer wieder ihr Interesse an Reformen in diesem Bereich deutlich gemacht. So ist derzeit ein Reformvorhaben in Verhandlung, das jedoch, so scheint es, nur auf den Austausch der durch die PiS protegierten Personen zielt. Tusks Partei Bürgerplattform (Platforma Obywatelska-PO) begründet ihren Reformvorschlag mit der Notwendigkeit, die politische Kontrolle auf die öffentlich-rechtlichen Medien (Fernsehen und Radio) einzuschränken. Nach dem Gesetzentwurf der $P O$ soll nicht mehr der KRRiT, sondern das Amt für Elektronische Kommunikation (Urząd Komunikacji Elektronicznej - UKE) für die Vergabe von Sendelizenzen zuständig sein. Das UKE untersteht nicht wie der KRRiT dem Parlament, sondern der Regierung. Der Leiter des UKE soll jedoch vom Parlament benannt werden. Das Vorhaben scheiterte bisher am Veto von Staatspräsident Lech Kaczyński. Darüber hinaus ist ein weiterer Kernpunkt für die $P O$ die Abschaffung der Rundfunkgebühren. Stattdessen soll ein Fonds, der vom polnischen Staatshaushalt getragen wird, die öffentlich-rechtlichen Sender finanzieren.

Eine Reform des Pressegesetzes, die unter anderem die Vereinigung Polnischer Journalisten fordert, tritt vor dem Hintergrund dieser Diskussion in weite Ferne. Die im gültigen Mediengesetz enthaltenen Aussagen zur öffentlich-zivilgesellschaftlichen Rolle der Medien machen aus ihrer Sicht die Rolle der Medien als Gegengewicht zur Politik nicht ausreichend deutlich. Nicht zuletzt sind viele Fragen der journalistischen Praxis in Polen nicht beantwortet. So gibt es keine Berufsbeschreibung des Journalisten - die Frage, wer als Journalist tätig sein kann und wer nicht, ist ungeklärt, und auch die Interessenvertretung über die Journalistenvereinigung funktioniert nur teilweise. Viele Journa- 


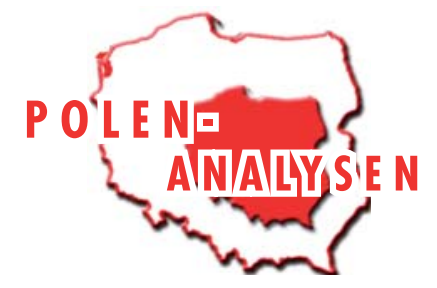

polen-analysen 50/09

listen gehören ihr nicht an und fühlen sich nicht an ihren Berufsstand gebunden und somit auch nicht den ethischen Vorgaben verpflichtet, die von der SDP über die Medienethikcharta vertreten werden. Die Unvollständigkeit des Pressegesetzes lässt also auch Fragen mit Blick auf die Journalisten offen -gehen sie mit der Freiheit verantwortungsbewusst um, die es ihnen lässt? Das Verhältnis von Politik und Medien ist schwierig und ambivalent, der rechtliche Rahmen erweist sich als umstritten und fragmentarisch.

\section{Akteurskonstellationen und Konfliktlinien}

Das wohl auffälligste Phänomen auf dem polnischen Pressemarkt ist die Spaltung der Qualitätszeitungen in ideologische Lager. Dieses Phänomen betrifft vor allem die beiden großen Tageszeitungen Gazeta Wyborcza und Rzeczpospolita. Auf der einen Seite steht die linksliberale Gazeta Wyborcza mit ihrem Chefredakteur Adam Michnik. Dieser vertrat mit seiner nach 1989 mit Abstand einflussreichsten Zeitung die Politik des »dicken Strichs" ("gruba kreska»). Diese besagte, dass das Kapitel der kommunistischen Ära abgeschlossen und möglichst nicht die Gesellschaft spaltend erneut geöffnet werden sollte, um der jungen Demokratie einen politisch unbelasteten Neuanfang zu ermöglichen. Die andere Seite kann als das "Kaczyński-Lager» beschrieben werden. Die Aufarbeitung der kommunistischen Geschichte Polens und die Aufdeckung von Verstrickungen im System gehört für dieses Lager zu den wichtigsten Aufgaben des Staates.

Nach ihrem Willen sollte daher auch innerhalb der Medien eine so genannte "lustracja» (»Lustration«, wörtl. Übersetzung: Prüfung, Revision) durchgeführt werden, in deren Rahmen alle Journalisten Rechenschaft über eine eventuelle informelle Zusammenarbeit mit den kommunistischen Sicherheitsdiensten ablegen sollten. Die heftige Auseinandersetzung, die daraufhin entbrannte, spielte sich auch in den Artikeln der beiden Qualitätszeitungen ab. Auch in anderen Politikbereichen vertreten die beiden Blätter deutlich divergierende Positionen. Während sich die Gazeta Wyborcza eine pro-integrative europäische Berichterstattung auf die Fahnen geschrieben hat, wird in der Rzeczpospolita über die Wahrung nationaler Werte gestritten und den Meinungen viel Raum gegeben, für die die Europäische Union nicht mehr als eine wirtschaftliche Interessengemeinschaft darstellt.

Dies alles macht deutlich, dass polnische Printmedien und damit polnische Journalisten vergleichsweise stark als Akteure in den politischen Diskurs eingebunden sind. Das Gefühl der Zugehörigkeit zu bestimmten ideologischen Lagern macht die Trennung zwischen Infor- mation und kommentierender Begleitung schwer möglich. Darüber hinaus sind einige Journalisten regelrechte Medienstars und spielen als Kommentatoren politischer Ereignisse und Experten eine wichtige gesellschaftliche Rolle. Nicht wenige Zeitungsjournalisten platzieren eigene Formate im Fernsehen oder Radio. Immer wieder wird Kritik laut, dass Journalisten ihre Aufgabe nicht als die eines Beobachters verstehen, sondern zu bewussten Akteuren des öffentlichen, politischen Lebens werden. Der bekannte Journalist Jacek Żakowski kritsierte, dass einige Journalisten mehr um ihre Medienpräsenz als um ihre journalistischen Qualitäten besorgt seien.

Die Vermischung von Politik und Medien ist nicht zuletzt seit »Rywingate», der Rywin-Affaire, ein wichtiges Thema für die polnische Presse. Ausgangspunkt war eine Änderung des Rundfunkgesetzes im Frühjahr 2002 unter der Regierung von Leszek Miller (Demokratische Linksallianz, Sojusz Lewicy Demokratycznej$S L D)$, die die Stellung der öffentlich-rechtlichen Medien sowie die des Rundfunkrates gegenüber den privaten Medien erheblich stärkte, was von heftigen Auseinandersetzungen zwischen dem Lager der Privaten und der Regierung begleitet wurde. Im selben Jahr machte der TVP1-Filmproduzent Lew Rywin dem Chefredakteur der Gazeta Wyborcza Adam Michnik das Angebot, der Medienkonzern Agora S.A., der u. a. die Gazeta Wyborcza herausgibt, könne den zweiten polnischen Kanal TVP2 gegen die Zahlung von rund 17 Mio. Dollar übernehmen. Michnik zeichnete das Gespräch auf und brachte es an die Öffentlichkeit. Eine Katastrophe für die Medien, welche zusehends Ansehen und Glaubwürdigkeit in der Bevölkerung verloren. Die Affäre und die politischen Verstrickungen sind bis heute nicht vollständig aufgeklärt. Bestärkt wurde dabei der in Polen verbreitete Verdacht, dass der Staat auf dem Medienmarkt doch sehr viel mehr seine Finger im Spiel hat als angenommen.

Eine weitere entscheidende Zäsur für den polnischen Medienmarkt war das Auftreten von TVN24 und Fakt zu Anfang des Jahrzehnts. Seitdem lässt sich die zunehmende Boulevardisierung der polnischen Medien illustrieren. Ein wichtiger Indikator für den Einfluss der Tageszeitung Fakt auf dem polnischen Markt ist die Kopie ihres Formats mit der zweiten Boulevardzeitung am Markt, Super Express, und nicht zuletzt zeigen die Auflagenzahlen, dass mit dem Markteintritt von Fakt die Tageszeitungen einen erheblichen Anteil der Leser an Fakt abtreten mussten. Ein weiterer Hinweis auf Boulevardisierung ist, dass die größte und beständig wachsende Gruppe von Printerzeugnissen die Frauen- und Männerzeitschriften sowie Hobbymagazine sind. Im 


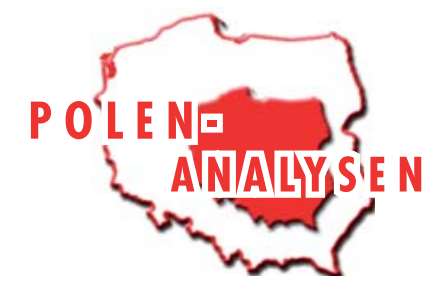

Kampf um Auflagenzahlen und Leser, so zeigt sich auch in Polen, zählen Bilder, Skandale und Emotionen. Auch im politischen Leben ist zu beobachten, dass Politiker ihr Handeln an solche Formate anpassen. Erst kürzlich verordnete sich die Regierung unter Ministerpräsident Tusk eine Gehaltskürzung - das Thema Politikergehälter gehört u. a. zu den beliebtesten der Zeitung Fakt.

\section{Fazit}

Ein Befund bei der Analyse der polnischen Presselandschaft ist, dass sich Strukturen entwickelt haben, die auch in westeuropäischen Ländern bekannt sind. Die polnische Presselandschaft hat seit dem »Runden Tisch" gewaltige Veränderungen erlebt. Seit der Öffnung des Pressemarktes sind in Polen mindestens so viele Zeitungen neu erschienen und wieder von der Bildfläche verschwunden wie politische Parteien - unüberschaubar viele. Mittlerweile hat sich das Angebot an politischer Tages- und Wochenpresse stabilisiert und sich eine gewisse Pluralisierung des Angebots etabliert. Dazu haben neue Titel aus dem Springer-Konzern (Newsweek, Fakt, Dziennik) nicht unerheblich beigetragen. Die Probleme, mit denen die Presse zu kämpfen hat, haben große Ähnlichkeit mit den aus dem Westen bekannten: zurückgehende Auflagenzahlen der Tages- und Wochenpresse, besonders der Qualitätspresse, Boulevardisierung der Inhalte und Medialisierung der Journalisten.

Ein zweiter Befund zeigt sich mit Blick auf die wichtigsten inhaltlichen Konfliktlinien. Die polnische Presse ist, nicht zuletzt im Umgang mit der kommunistischen Vergangenheit Polens, inhaltlich tief gespalten. Hierbei spielen die Journalisten selbst eine bedeutende Rolle, indem sie aktiver als zum Beispiel in Deutschland üblich als Akteure an politischen Debatten teilnehmen und ihre Positionen offensiv präsentieren. Besonders die Frage der »lustracja«, der Offenlegung von Akten inoffizieller Mitarbeiter des Sicherheitsdienstes der Volksrepublik Polen, aber auch andere Affären und Konflikte haben die Spaltung in unterschiedliche Lager offengelegt, die ihre Kämpfe gern auch über die Presse austragen.

Bei diesem zweiten Befund zeigt sich also, dass die Grenzen zwischen Politik und Medien fließend sind. Gelingt Polens Presse eine Transformation des Selbstverständnisses, die der Entwicklung hin zu einer demokratische Zivilgesellschaft tatsächlich entspricht? Nicht zuletzt das im Unklaren verbleibende Presserecht und erhebliche ideologische Auseinandersetzungen in den Reihen der Journalisten führen die Schwierigkeiten auf dem Weg dorthin vor Augen. Möglicherweise ist Polen aber auch ein Beispiel für eine Entwicklung, auf die auch die westliche Presse zusteuert: Rationalisierte Berichterstattung, sinkende Leserzahlen und intensiver Sensationsjournalismus weisen kaum den Weg zu einer Zivilgesellschaft, die in der Presse ein zuverlässiges Medium hat.

\section{Über die Autorin}

Johanna Möller, Jahrgang 1980, ist wissenschaftliche Mitarbeiterin im Forschungsprojekt "The Transnationalization of Public Spheres in Europe« (Leitung: Prof. Dr. Andreas Hepp) im Sonderforschungsbereich 597 »Transformations of the State« an der Universität Bremen und der Jacobs University Bremen. Das Forschungsprojekt untersucht die Europäisierung nationaler Öffentlichkeiten in sechs europäischen Ländern, darunter Polen. Nach dem Studium der Politikwissenschaft an der FU Berlin und UJ Krakau promoviert Johanna Möller derzeit im Bereich Kommunikationswissenschaften an der Jacobs University zum Thema »Transkulturelle Öffentlichkeitsakteure in den deutsch-polnischen Beziehungen«. 


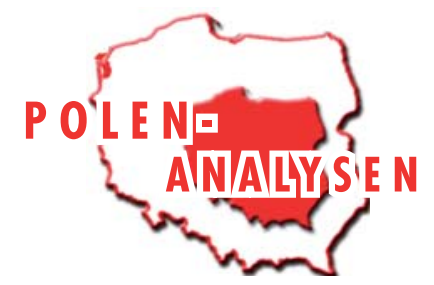

\section{Tabellen und Grafiken}

\section{Auflagen und Verkauf polnischer Printmedien}

Durchschnittliche Einzelauflage - Überregionale Tageszeitungen 2000-2008

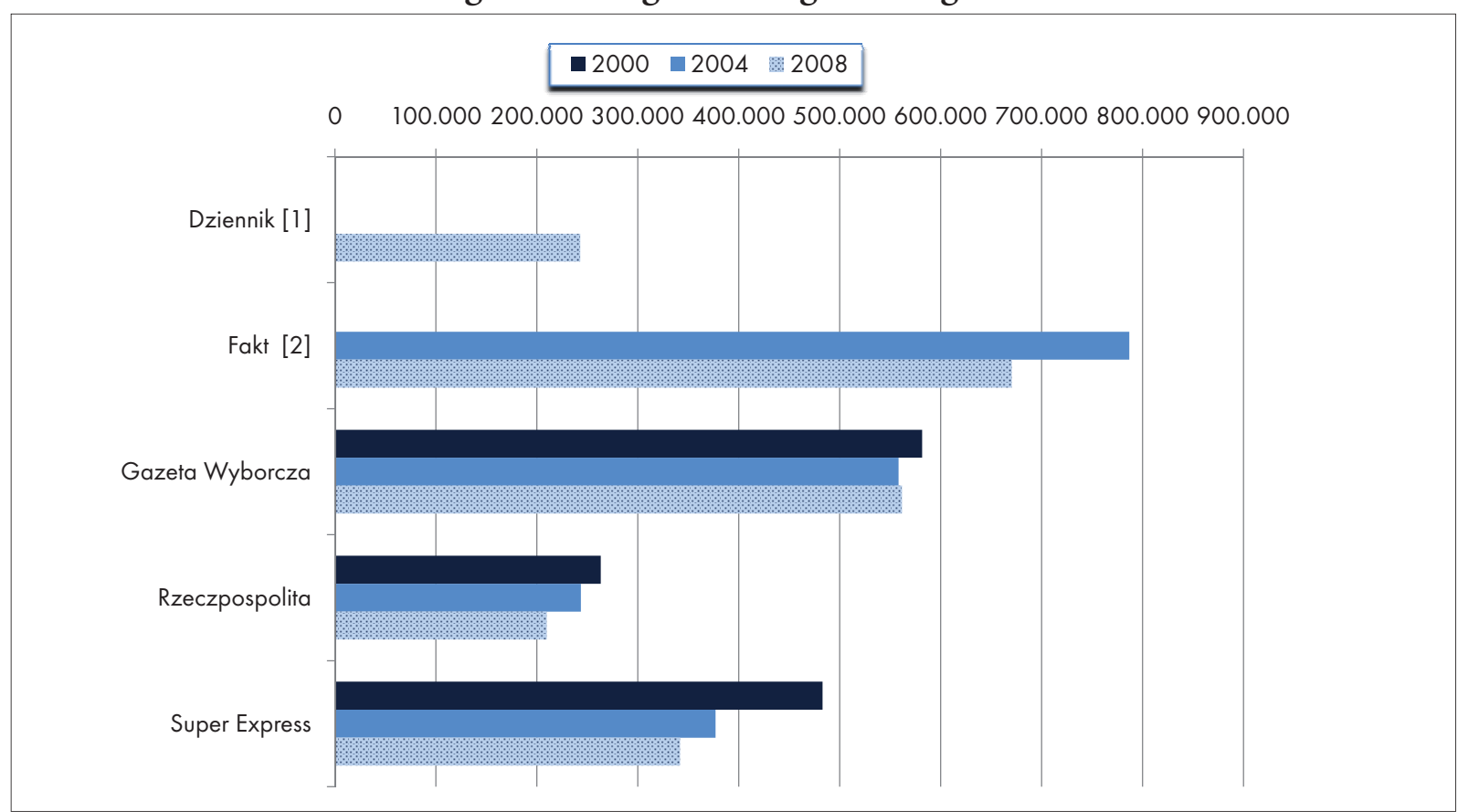

Durchschnittliche Anzahl der verkauften Exemplare - Überregionale Tageszeitungen 2000-2008

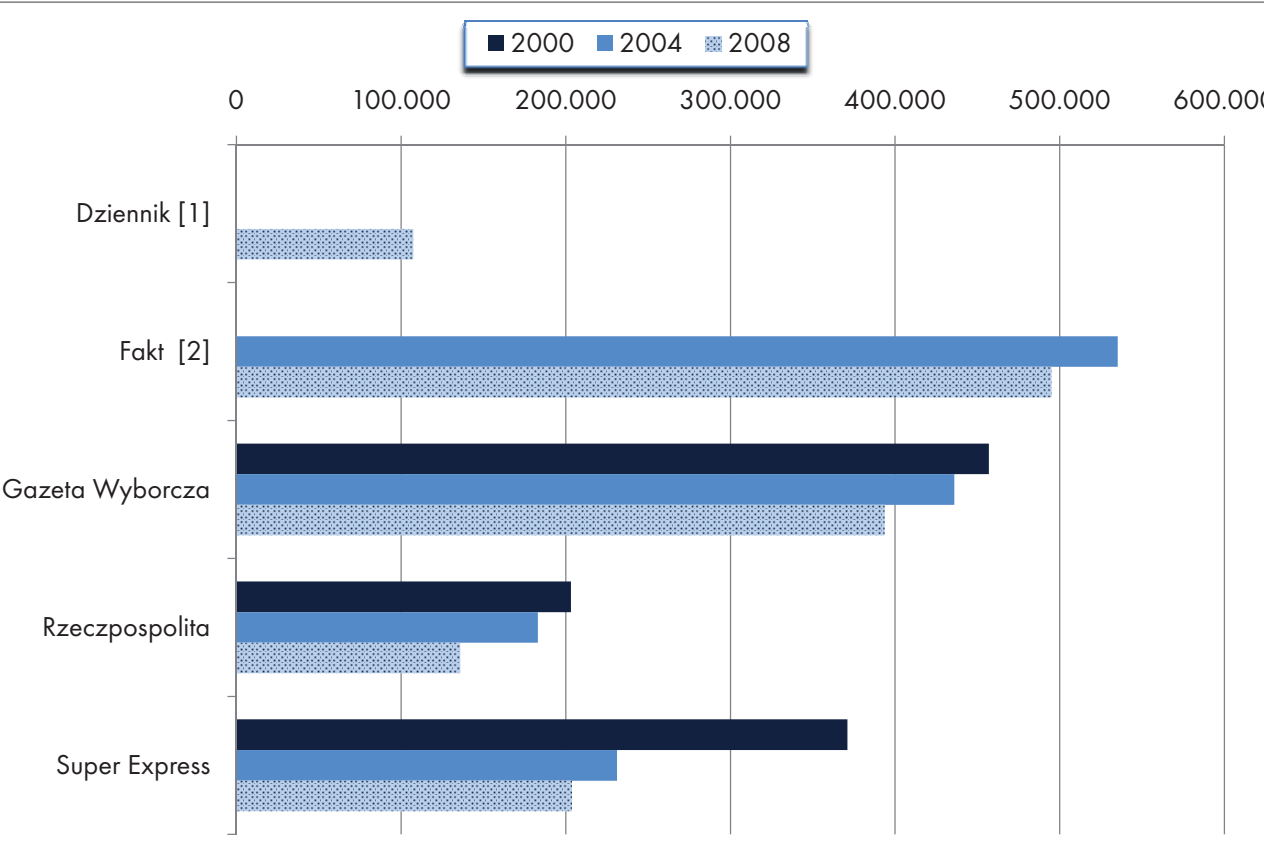

[1] - erscheint seit 2006; [2] - erscheint seit 2003; Durchschnittliche Einzelauflage: durchschnittliche Anzahl der Exemplare einer Ausgabe pro Jahr, d.h Gesamtauflage der Zeitung im Jahr x dividiert durch die Anzahl der Ausgaben im Jahr x; Durchschnittliche Anzahl der verkauften Exemplare (Einzelverkauf, Abonnement usw.), ermittelt wie oben; für die zugrundeliegenden Zablen siehe Tabelle aufS. 11.

Quelle: Komunikaty Zarzadu Zwiazku Kontroli Dystrybucji Prasy o uysokości naktadów i dystrybucji pism kontrolowanych przez ZKDP w 2000 i 2004 roku; Publikacje danych z deklaracji wydawców dzienników i tygodników kontrolowanych przez ZKDP za styczeń-grudzień 2008. 


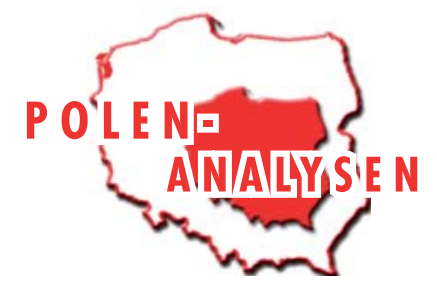

Durchschnittliche Einzelauflage - Regionale Tageszeitungen 2000-2008

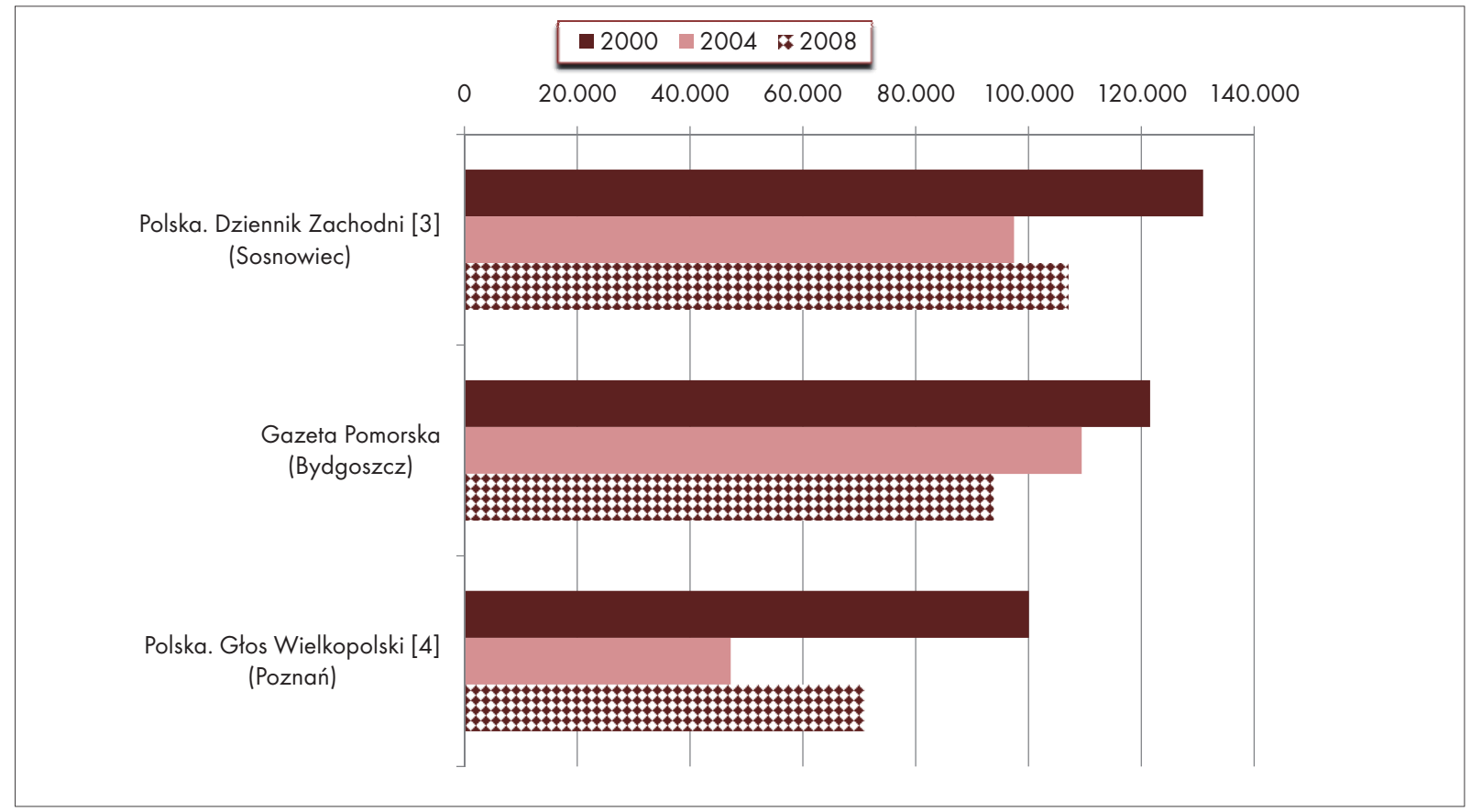

Durchschnittliche Anzahl der verkauften Exemplare - Regionale Tageszeitungen 2000-2008

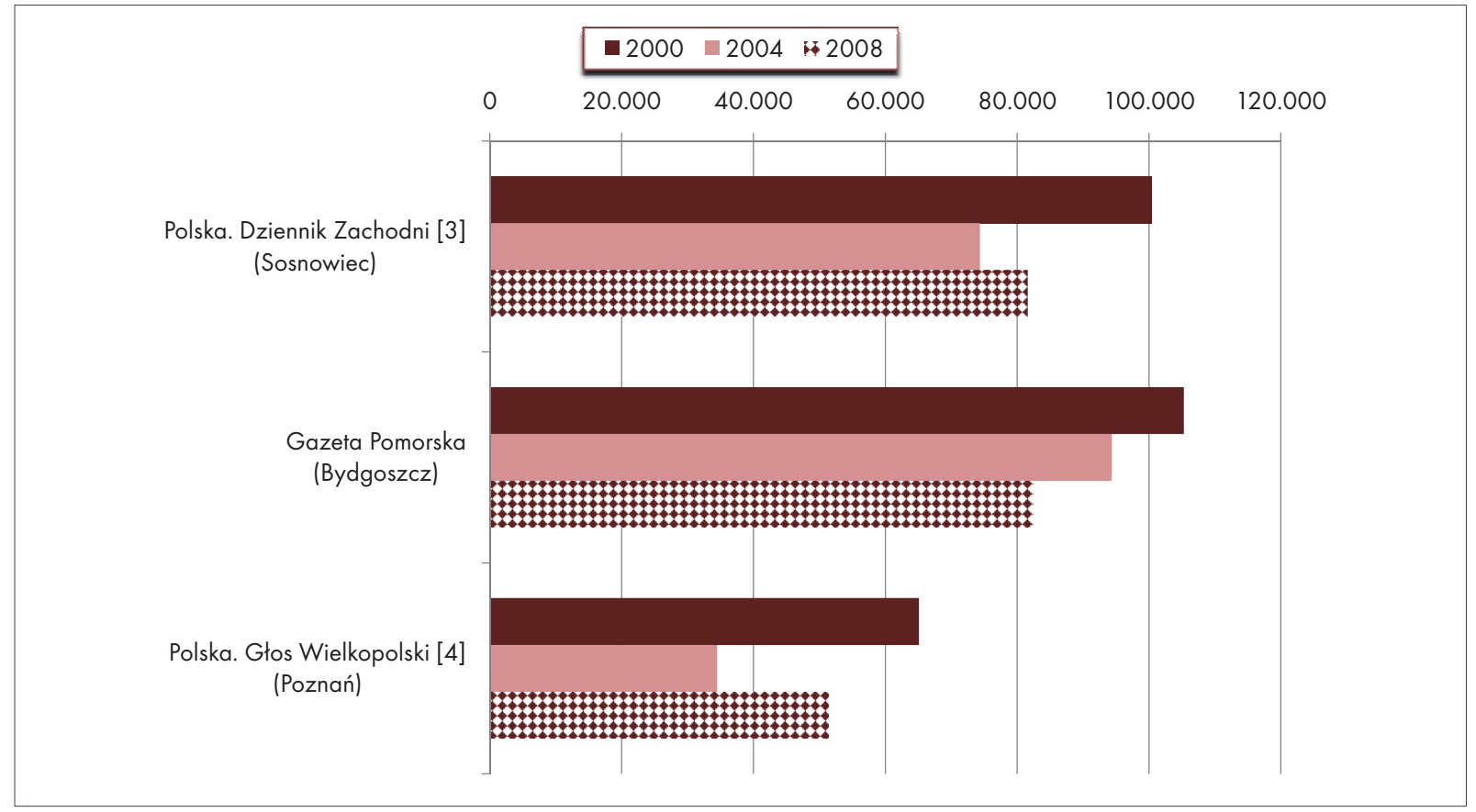

[3] - als Dziennik Zachodni bis 2007; [4] - als Gtos Wielkopolski bis 2007; Durchschnittliche Einzelauflage: durchschnittliche Anzabl der Exemplare einer Ausgabe pro Jahr, d.h Gesamtauflage der Zeitung im Jahr x dividiert durch die Anzahl der Ausgaben im Jahr x; Durchschnittliche Anzahl der verkauften Exemplare (Einzelverkauf, Abonnement usw.), ermittelt wie oben; für die zugrundeliegenden Zablen siehe Tabelle aufS. 11.

Quelle: Komunikaty Zarzadu Związu Kontroli Dystrybucji Prasy o uysokości naktadów i dystrybucji pism kontrolowanych przez ZKDP $w 2000$ i 2004 roku; Publikacje danych z deklaracji uydawców dzienników i tygodników kontrolowanych przez ZKDP za styczeń-grudzień 2008. 


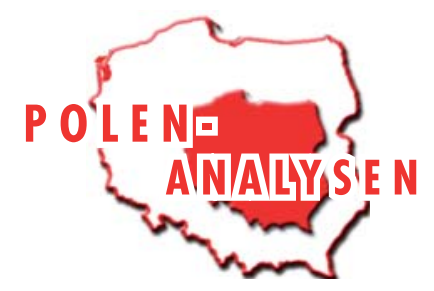

Durchschnittliche Einzelauflage - Gesellschaftspolitische Wochenmagazine 2000-2008

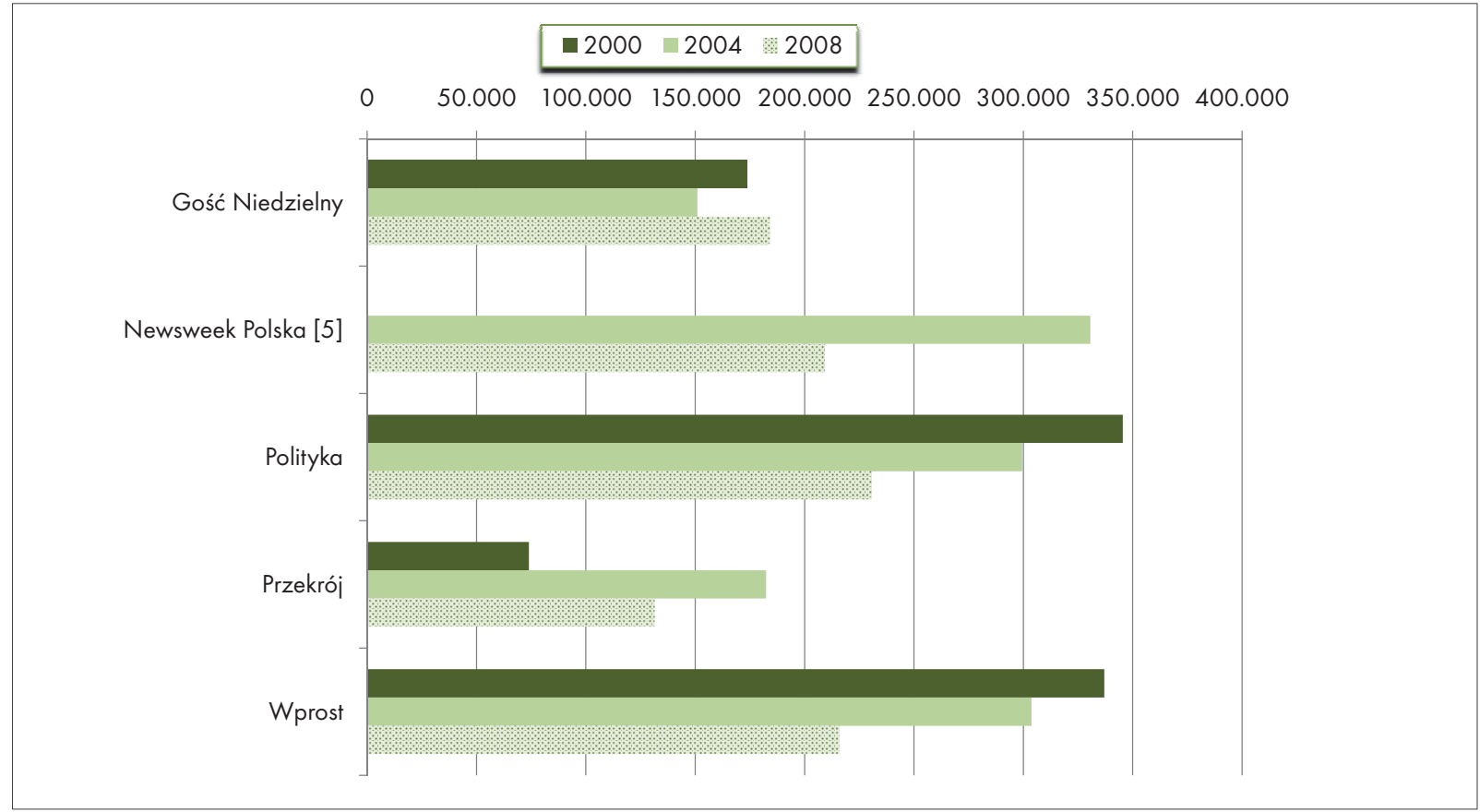

Durchschnittliche Anzahl der verkauften Exemplare - Gesellschaftspolitische Wochenmagazine 2000-2008

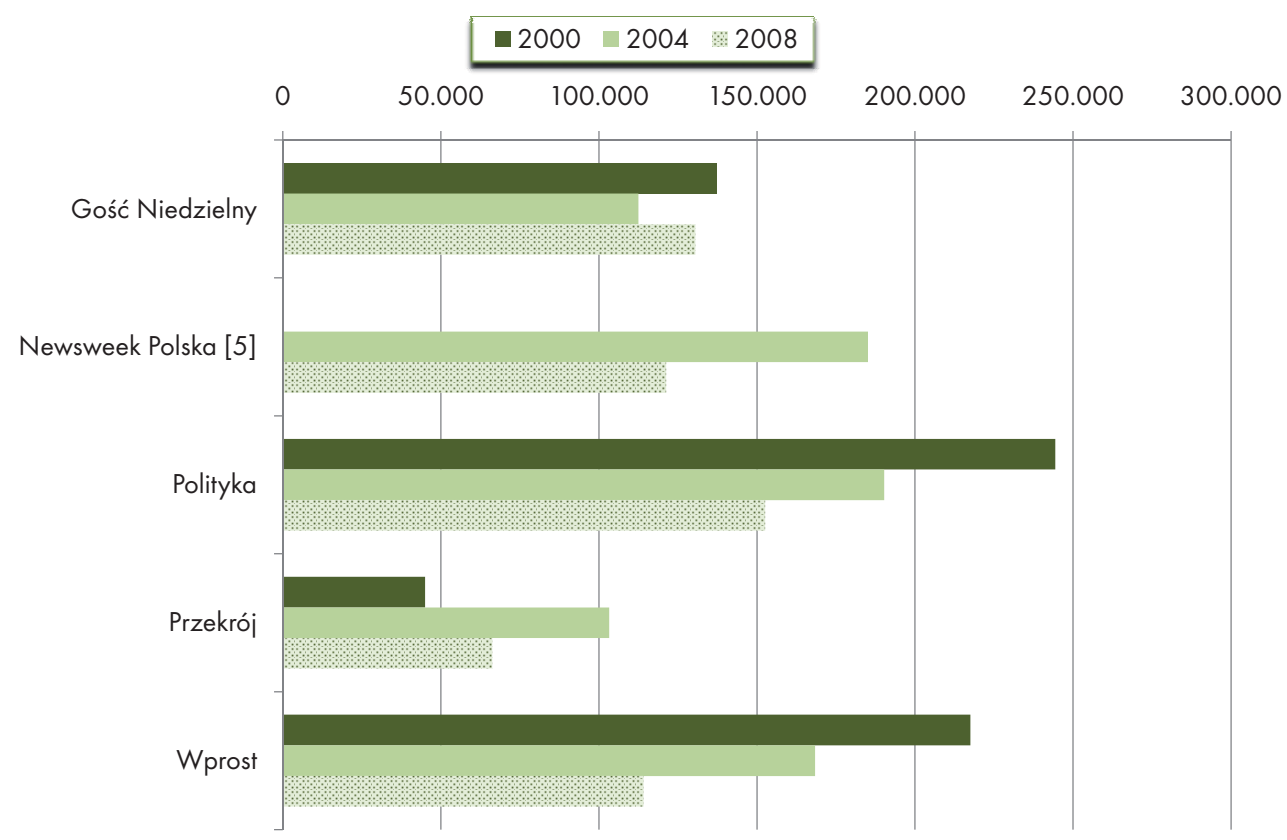

[5] - erscheint seit 2001; Durchschnittliche Einzelauflage: durchschnittliche Anzahl der Exemplare einer Ausgabe pro Jahr, d.h Gesamtauflage der Zeitung im Jahr x dividiert durch die Anzahl der Ausgaben im Jahr x; Durchschnittliche Anzahl der verkauften Exemplare (Einzelverkauf, Abonnement usw.), ermittelt wie oben; für die zugrundeliegenden Zablen siehe Tabelle aufS. 11.

Quelle: Komunikaty Zarzadu Zwiazku Kontroli Dystrybucji Prasy o wysokości naktadów i dystrybucji pism kontrolowanych przez ZKDP w 2000 i 2004 roku; Publikacje danych z deklaracji wydawców dzienników i tygodników kontrolowanych przez ZKDP za styczeń-grudzień 2008. 


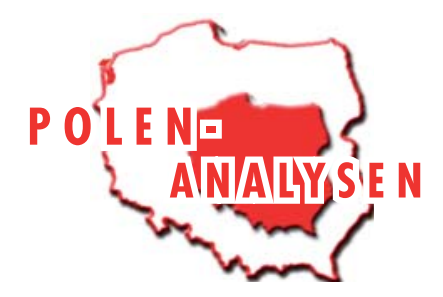

polen-analysen 50/09

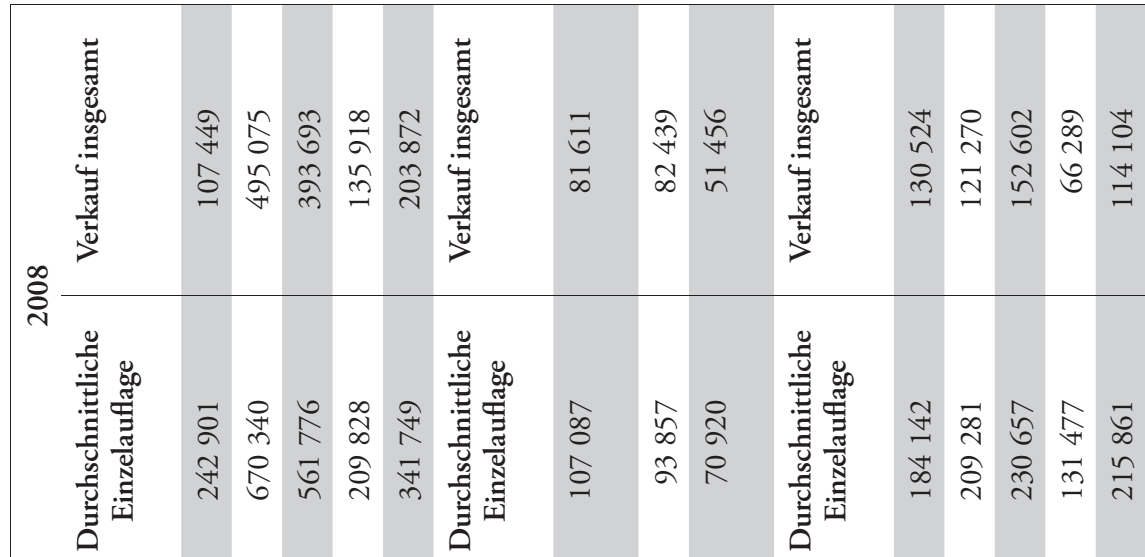

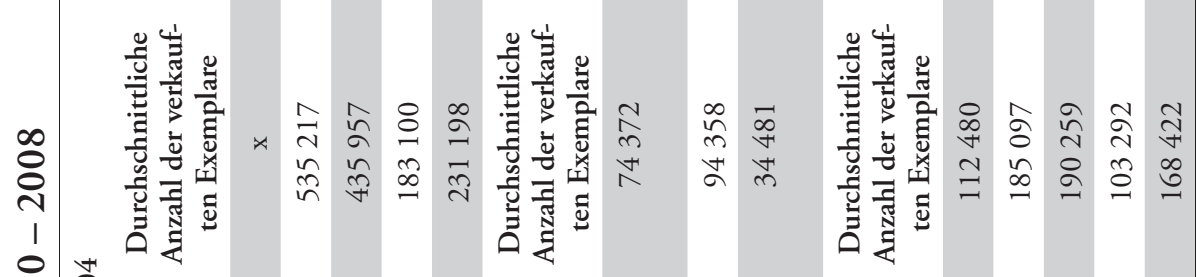

8

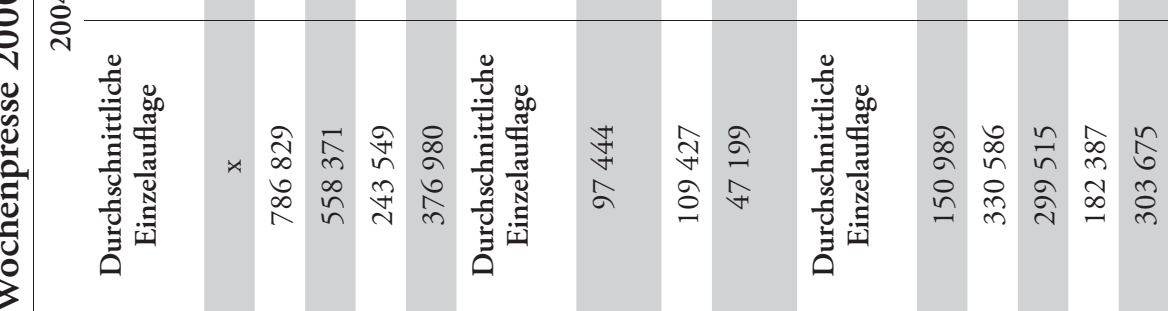

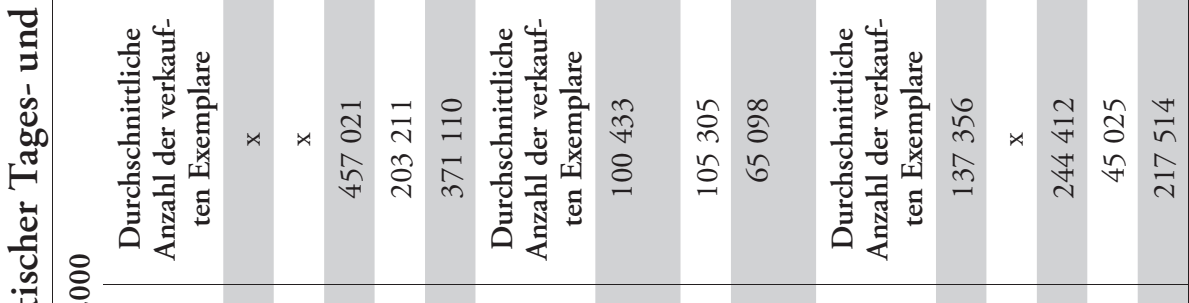

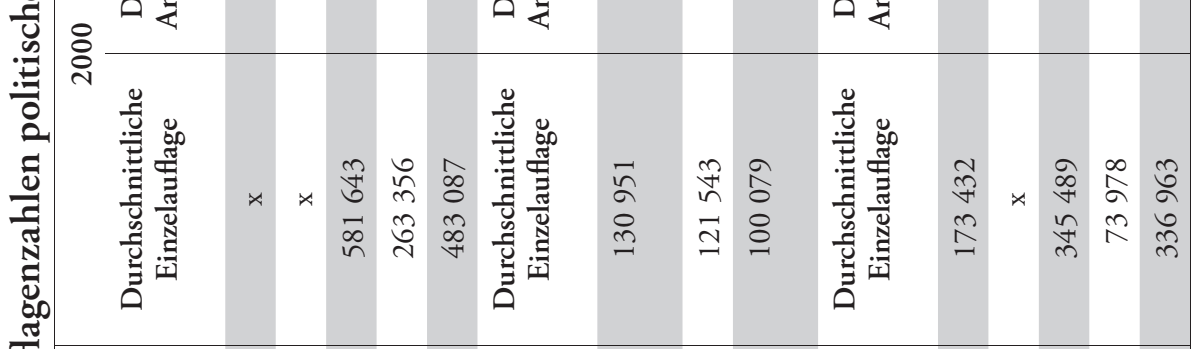

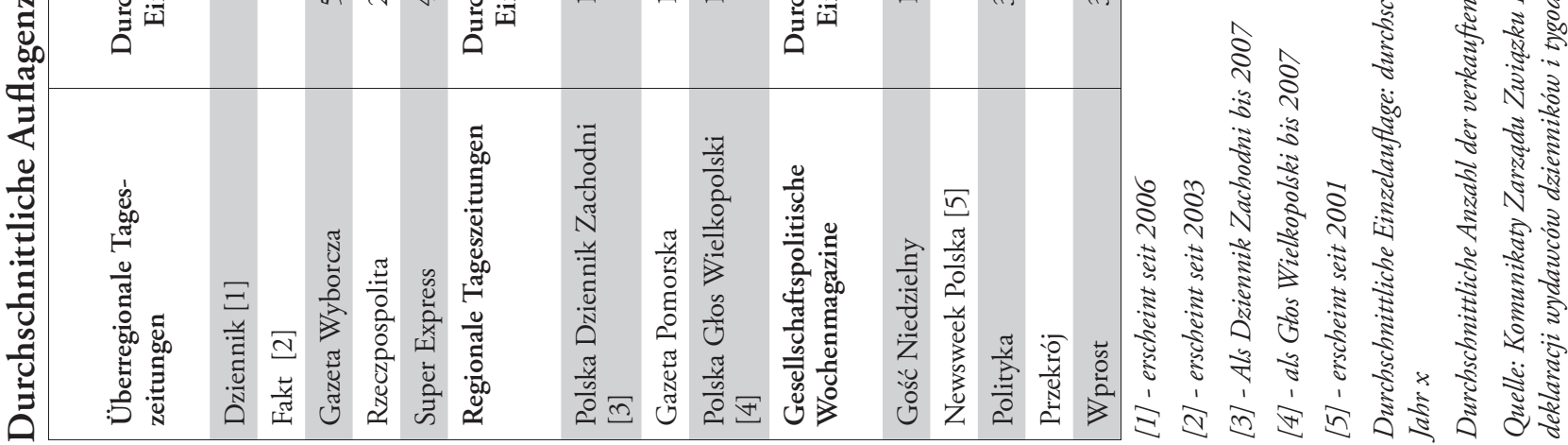




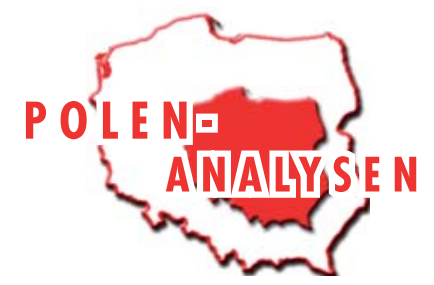

Überregionale Tageszeitungen: Auflagenhöhe 2008-2009

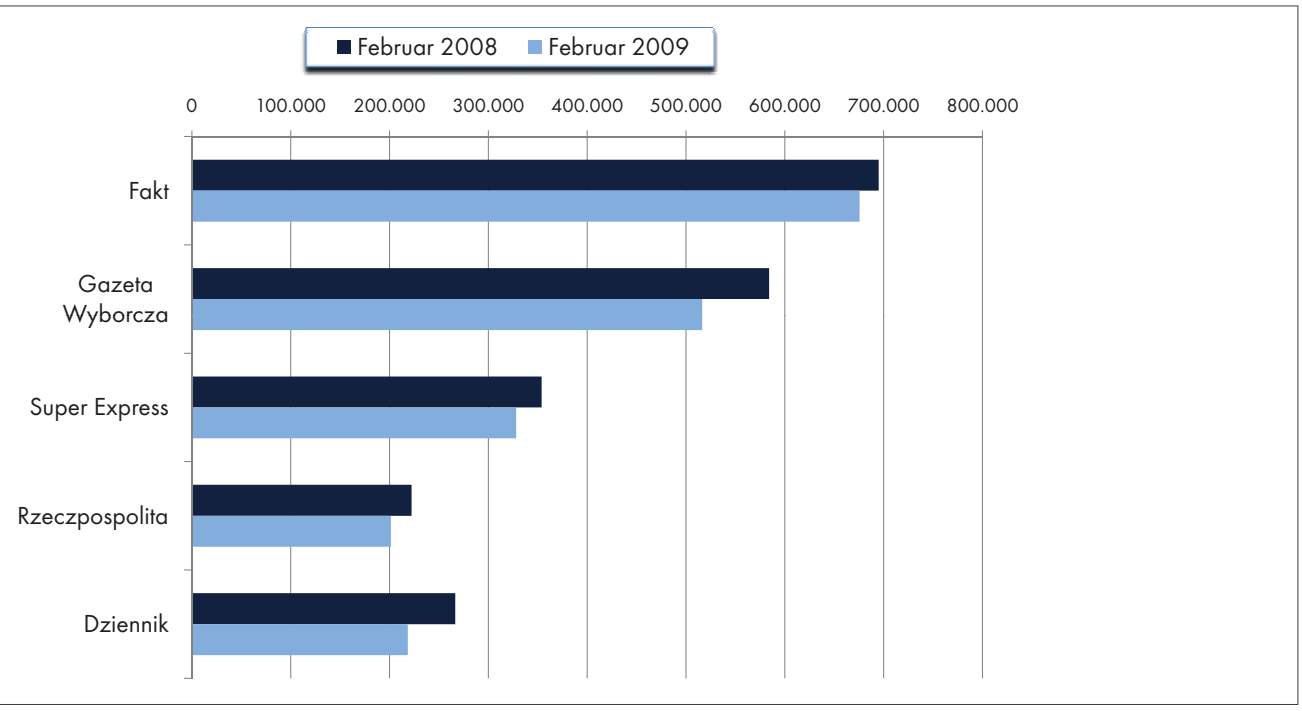

Überregionale Tageszeitungen: verkaufte Exemplare 2008-2009

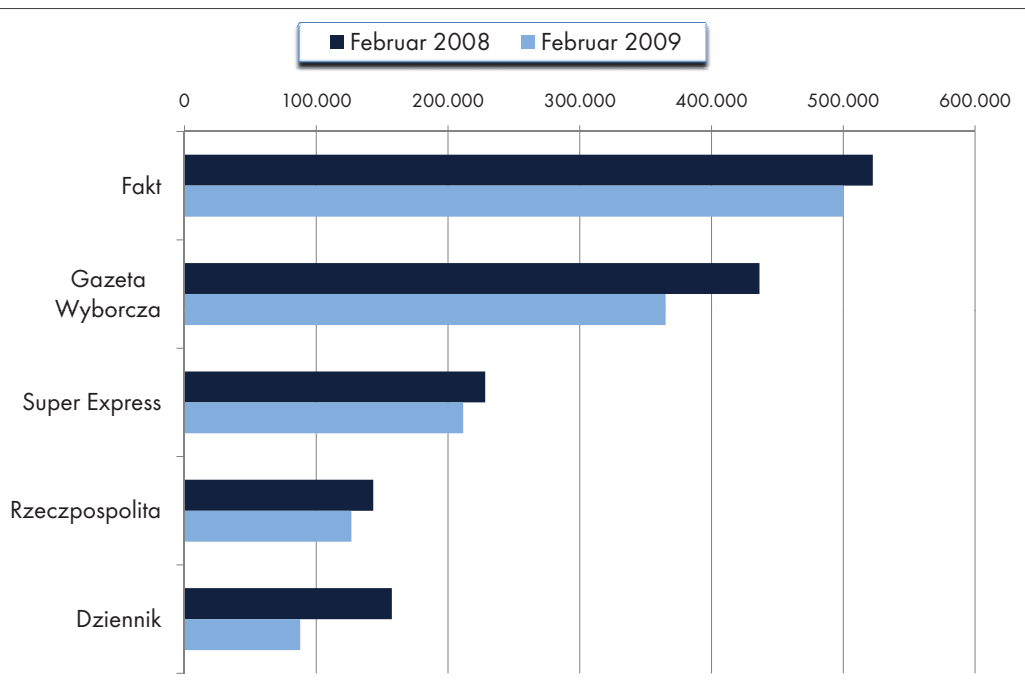

Überregionale Tageszeitungen: Auflagenhöhe und verkaufte Exemplare 2008-2009

\begin{tabular}{|l|c|c|c|c|}
\hline & \multicolumn{2}{|c|}{ Februar 2008 } & \multicolumn{2}{c|}{ Februar 2009 } \\
& $\begin{array}{c}\text { Höhe der Einzelauflage } \\
\varnothing\end{array}$ & Verkauf & Höhe der Einzelauflage & $\emptyset$ \\
Fakt & 694.998 & 522.396 & 675.588 & 500.066 \\
\hline Gazeta Wyborcza & 584.066 & 436.410 & 516.466 & 365.209 \\
\hline Super Express & 353.833 & 228.250 & 328.204 & 211.497 \\
\hline Rzeczpospolita & 222.300 & 143.208 & 201.371 & 126.623 \\
\hline Dziennik & 266.494 & 157.352 & 218.412 & 87.871 \\
\hline
\end{tabular}

Quelle: www.wirtualnemedia.pl/print.htm? article=2678420 (abgerufen am 14.04.2009) 


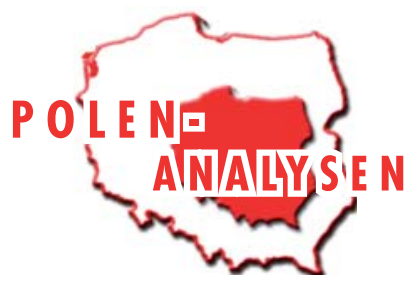

Wochenzeitungen: Auflagenhöhe 2008-2009

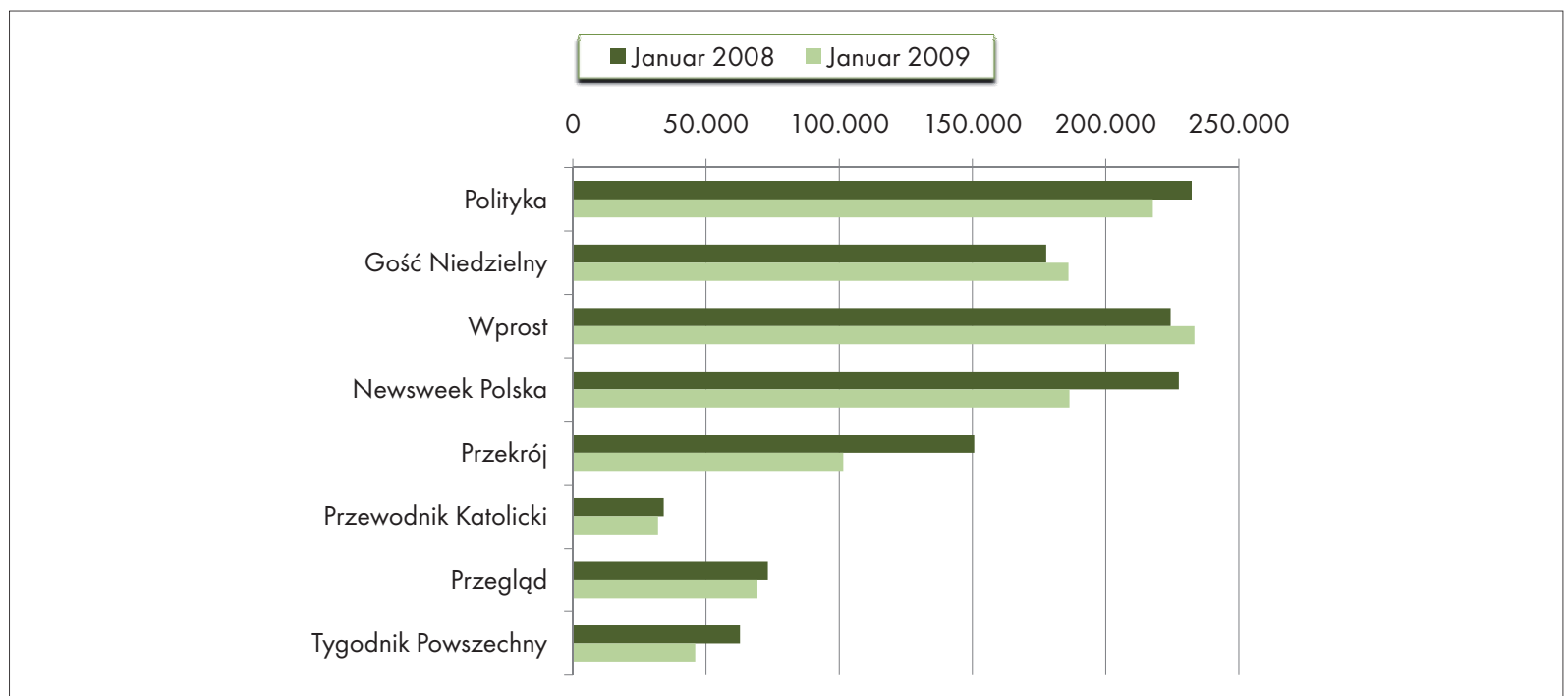

Wochenzeitungen: verkaufte Exemplare 2008-2009

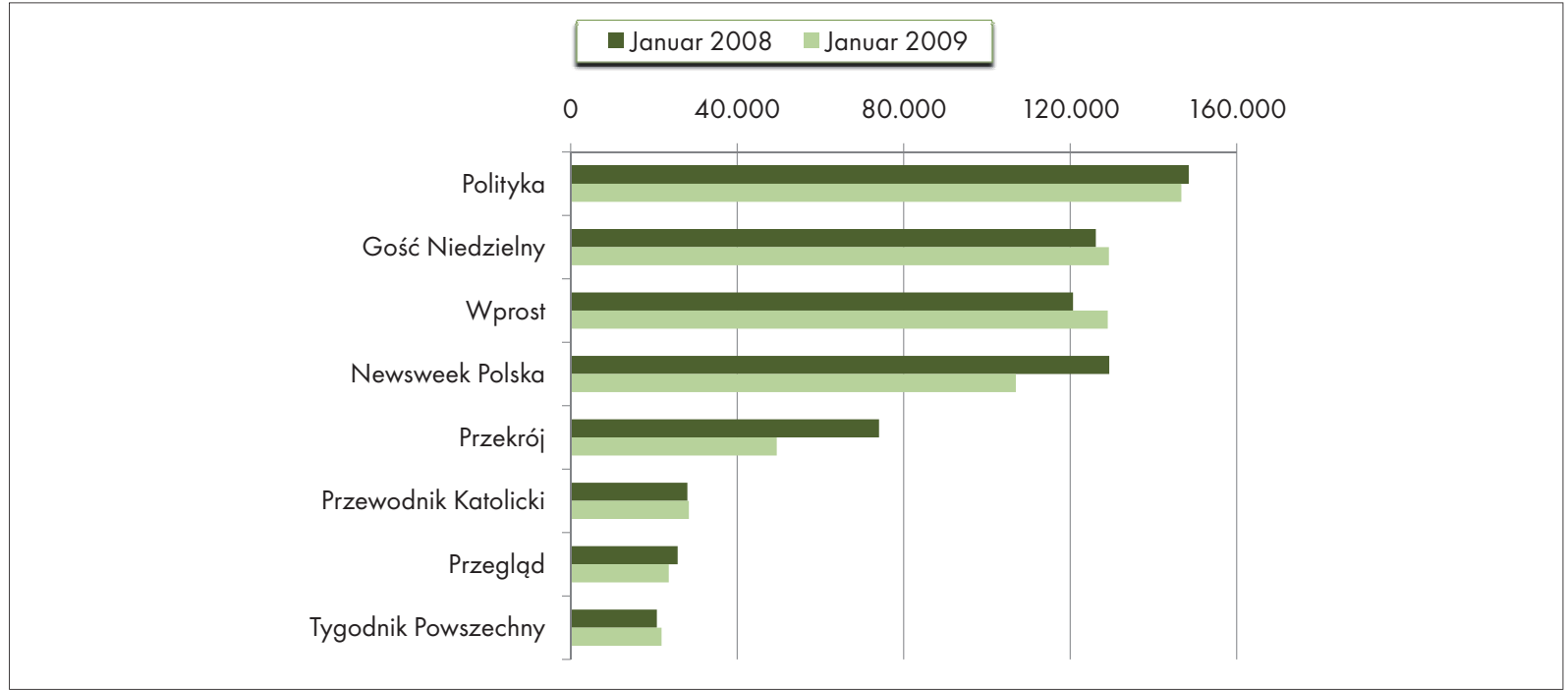

Wochenzeitungen: Auflagenhöhe und verkaufte Exemplare 2008-2009

\begin{tabular}{|l|c|c|c|c|}
\hline & \multicolumn{2}{|c|}{ Januar 2008 } & \multicolumn{2}{c|}{ Januar 2009 } \\
& $\begin{array}{c}\text { Höhe der Einzelauflage } \\
\varnothing\end{array}$ & Verkauf & Höhe der Einzelauflage & $\varnothing$ \\
\hline Polityka & 232.160 & 148.472 & 217.714 & 146.704 \\
\hline Gość Niedzielny & 177.661 & 126.149 & 186.034 & 129.290 \\
\hline Wprost & 224.384 & 120.677 & 233.319 & 129.010 \\
\hline Newsweek Polska & 227.445 & 129.367 & 186.415 & 106.930 \\
\hline Przekrój & 150.656 & 74.098 & 101.511 & 49.504 \\
\hline Przewodnik Katolicki & 34.088 & 28.020 & 31.966 & 28.381 \\
\hline Przegląd & 73.200 & 25.700 & 69.300 & 23.551 \\
\hline Tygodnik Powszechny & 62.778 & 20.701 & 45.917 & 21.836 \\
\hline
\end{tabular}

Quelle: www. wirtualnemedia.pl/print.htm? article=2665151 (abgerufen am 14.04.2009) 


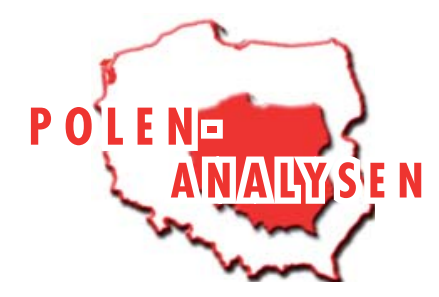

Chronik

\section{Vom 07. bis zum 20. April 2009}

07.04.2009 $\quad$ Nach Angaben des Ministeriums für Arbeit und Sozialpolitik betrug die Zahl der Arbeitslosen Ende März 1,76 Mio., was einen Anstieg um 2,3 \% im Vergleich zu Ende Februar bedeutet.

08.04.2009 Während seines eintägigen Besuchs in Afghanistan trifft sich Staatspräsident Lech Kaczyński in Kabul mit Staatspräsident Hamid Karzai und mit Soldaten des polnischen Kontingents in Ghazni. Neben einer Erhöhung des Kontingents stellt Kaczyński eine Aufstockung der humanitären Hilfe in Aussicht.

09.04.2009 Staatspräsident Lech Kaczyński unterzeichnet den Regierungsantrag auf Erhöhung des polnischen Kontingents in Afghanistan von derzeit 1.600 auf 2.000 Soldaten. Darüber hinaus sollen weitere 200 Soldaten in Polen für kurzfristige Einsätze in Afghanistan vorbereitet werden. Die Erhöhung des Kontingents wird mit wachsenden Gefahren vor den für August geplanten afghanischen Präsidentschaftswahlen begründet.

10.04.2009 Sejmmarschall Bronisław Komorowski nimmt in Katyn an einer Trauerfeier zum Gedenken an die mehr als 4.000 ermordeten polnischen Offiziere teil, die dort 1940 vom sowjetischen Geheimdienst NKWD ermordet worden waren. Neben Angehörigen der Ermordeten sind u. a. auch der stellvertretende Senatsmarschall Zbigniew Romaszewski, der letzte polnische Präsident im Londoner Exil, Ryszard Kaczorowski, und der Militärbischof der Polnischen Armee, Tadeusz Płoski, anwesend.

11.04.2009 Die regierende Bürgerplattform (Platforma Obywatelska - PO) kündigt unter dem Motto "500 Treffen zu 500 Regierungstagen" eine Informationskampagne in ganz Polen an, in deren Rahmen die vergangenen 500 Tage Regierungstätigkeit zusammengefasst werden und Bürgerfragen beantwortet werden sollen.

13.04.2009 Zum Gedenken an die mehr als 20 Todesopfer eines Brands in einer Obdachlosenunterkunft in Kamień Pomorski (Cammin) ordnet Staatspräsident Lech Kaczyński eine dreitägige Staatstrauer an.

14.04.2009 Auf einer Pressekonferenz informieren Ministerpräsident Donald Tusk und Finanzminister Jacek Rostowski, dass Polen sich beim Internationalen Währungsfonds (IWF) um eine flexible Kreditlinie in Höhe von 20,5 Mrd. Dollar bemühen wolle. Tusk stellt heraus, dass der IWF Polen das Kreditangebot gemacht habe und Polen beabsichtige, es anzunehmen. Rostowski betont, Polen werde vom IWF innerhalb der Region als stabil beurteilt. Mit der Summe sollen die wirtschaftliche Führungsposition Polens in Mittel- und Osteuropa gestärkt und ausländische Investoren zu weiteren Investitionen angeregt werden. Die Mittel würden nicht in den Haushalt eingehen, sondern die Reserven der Polnischen Nationalbank (Narodowy Bank Polski - NBP) um ein Drittel aufstocken.

15.04.2009 Zur Eröffnung des Europäischen Wirtschaftskongresses in Kattowitz spricht sich Ministerpräsident Donald Tusk dagegen aus, die Wirtschaftskrise durch die Erhöhung der öffentlichen Ausgaben zu bekämpfen. Es wäre zynisch, die öffentlichen Mittel zu verschleudern und dies als Antikrisenmaßnahme darzustellen, so Tusk.

16.04.2009 Nach Angaben von Eurostat ist die polnische Industrieproduktion im Februar 2009 im Vergleich zu Februar 2008 um 12,3\% gefallen. In der Eurozone fiel sie um 17,5 \%, in Deutschland um mehr als $20 \%$.

17.04.2009 Nach der neuesten Umfrage des Zentrums zur Erforschung der öffentlichen Meinung (Centrum Badania Opinii Społecznej - CBOS) bewerten $44 \%$ der Befragten die Arbeit der Regierung von Ministerpräsident Donald Tusk als positiv, $26 \%$ als negativ, $27 \%$ äußern sich gleichgültig. $51 \%$ geben an, zufrieden mit der Tätigkeit von Ministerpräsident Donald Tusk zu sein, $36 \%$ sind unzufrieden, $14 \%$ geben keine Wertung. Vertrauen in die Wirtschaftspolitik der Regierung zu haben deklarieren $32 \%$ der Befragten, $53 \%$ äußern sich skeptisch, ob die Regierungspolitik zur Verbesserung der wirtschaftlichen Situation beitragen werde, $15 \%$ enthalten sich einer Meinung.

18.04.2009 Auf der Tagung des Politischen Rats von Recht und Gerechtigkeit (Prawo i Sprawiedliwość - PiS) in Warschau wird der Wahlkampf für die Wahlen zum Europäischen Parlament am 7. Juni eröffnet. Der Parteivorsitzende Jarosław Kaczyński kritisiert die Regierung von Ministerpräsident Donald Tusk, nicht ausreichend auf die Wirtschaftskrise zu reagieren, und unterstreicht, dass die PiS die einzige Partei sei, die sich um die polnische Landbevölkerung kümmere. Deren Interessen werde sie auch in der Europäischen Union vertreten. Den Medien wirft er vor, gegen die Opposition eingestellt zu sein und ihr keine Chance einzuräumen.

18.04.2009 Die Demokratische Linksallianz (Sojusz Lewicy Demokratycznej - SLD) und die Arbeitsunion (Unia Pracy - UP) eröffnen in Posen auf ihrem Konvent die Wahlkampagne für die Wahlen zum Europäischen Parlament am 7. Juni. Der SLD-Vorsitzende Grzegorz Napieralski spricht sich für ein starkes, geeintes und soziales Europa aus. Er kritisiert die Regierung und die oppositionelle Recht und Gerechtigkeit (Prawo i Sprawiedliwość - PiS) heftig und unterstreicht, dass die SLD den Beitritt Polens zur Europäischen Union ausgehandelt habe.

20.04.2009 Das Außenministerium teilt mit, dass Polen keinen Vertreter zur Anti-Rassismus-Konferenz der Vereinten Nationen in Genf entsenden werde. Grund seien berechtigte Befürchtungen, dass die Konferenz als Forum für unzulässige, insbesondere antisemitische Äußerungen genutzt werde, die nicht vom Respekt gegenüber anderen Rassen und Religionen geprägt seien. 


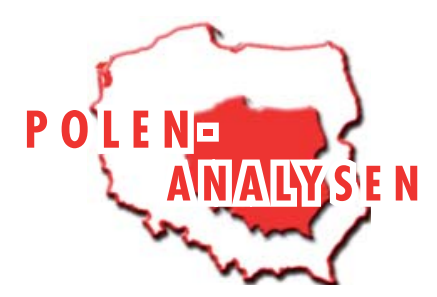

Über die Polen-Analysen

Die Polen-Analysen erscheinen zweimal monatlich als E-Mail-Dienst. Sie werden gemeinsam vom Deutschen PolenInstitut Darmstadt, der Bremer Forschungsstelle Osteuropa und der Deutschen Gesellschaft für Osteuropakunde herausgegeben.

Ein Archiv der Polen-Analysen finden Sie im Internet unter www.laender-analysen.de/polen

Kostenloses Abonnement unter http://www.deutsches-polen-institut.de/Newsletter/subscribe.php

\section{Deutsches Polen-Institut Darmstadt}

Das Deutsche Polen-Institut Darmstadt (DPI) ist ein Forschungs-, Informations-, und Veranstaltungszentrum für polnische Kultur, Geschichte, Politik, Gesellschaft und die deutsch-polnischen Beziehungen, die sich im Kontext der europäischen Integration entwickeln. Das seit März 1980 aktive und bis 1997 von Gründungsdirektor Karl Dedecius geleitete Institut ist eine Gemeinschaftsgründung der Stadt Darmstadt, der Länder Hessen und Rheinland-Pfalz sowie des Bundes. Seit 1987 ist die Trägerschaft auf die Kultusminister der Länder ausgedehnt. Einen wesentlichen Beitrag zur Verwirklichung der Institutsziele leisten private Stiftungen. Das DPI hat satzungsgemäß die Aufgabe, durch seine Arbeit zur Vertiefung der gegenseitigen Kenntnisse des kulturellen, geistigen und gesellschaftlichen Lebens von Polen und Deutschen beizutragen.

Ziel der Vermittlertätigkeit des DPI ist es, "die zu interessieren, auf die es politisch, wirtschaftlich, gesellschaftlich und kulturell im deutsch-polnischen Verhältnis ankommt« (Leitlinien 1997). Es geht um die Entscheider und Multiplikatoren in Politik, Kultur, Bildung, Verwaltung, Medien und Wirtschaft und, wesentlich stärker ausgeprägt als bisher, um das Hineinwirken in Wissenschaft, Forschung und Bildung.

Derzeit bemüht sich das DPI in Kooperation mit den verstreuten Orten wissenschaftlicher Polen-Kompetenz an deutschen Hochschulen und Forschungsinstituten verstärkt darum, ausgehend von einer Bestandsaufnahme deutscher Polen-Forschung Ort wissenschaftlicher Forschung und verbindendes, vernetzendes und kooperierendes Zentrum zu werden. Ausgangspunkt der Neuausrichtung ist die kaum mehr kontrollierbare Dynamik des Rückbaus der Ressourcen der wissenschaftlichen Polen-Kompetenz in den unterschiedlichen Disziplinen. Mit der über 55.000 Bände zählenden multidisziplinären Fachbibliothek für Polen mit einer einzigartigen Sammlung polnischer Literatur in der Originalsprache und in deutscher Übersetzung ist das DPI bereits ein geschätzter Ort der Recherche und des wissenschaftlichen Arbeitens. (www.deutsches-polen-institut.de)

\section{Forschungsstelle Osteuropa an der Universität Bremen}

1982 gegründet, widmet sich die Forschungsstelle Osteuropa an der Universität Bremen kulturellen und gesellschaftlichen Entwicklungen der Länder Ost- und Ostmitteleuropas in Zeitgeschichte und Gegenwart. Die Forschungsstelle besitzt in ihrem Archiv eine einzigartige Sammlung alternativer Kulturgüter und unabhängiger Texte aus den ehemaligen sozialistischen Ländern. Darunter befindet sich auch eine umfangreiche Sammlung des "Zweiten Umlaufs", die das Schrifttum und Dokumente unabhängiger Initiativen und gesellschaftlicher Gruppen in Polen aus der Zeit von 1976 bis zum Umbruch umfasst. Neben ausführlicher individueller Forschung zu Dissens und Gesellschaft im Sozialismus, leitet die Forschungsstelle seit Januar 2007 ein gemeinsames Projekt mit einem Verbund von internationalen Forschungsinstituten zum Thema »Das andere Osteuropa - die 1960er bis 1980er Jahre, Dissens in Politik und Gesellschaft, Alternativen in der Kultur. Beiträge zu einer vergleichenden Zeitgeschichte«, welches von der VolkswagenStiftung finanziert wird.

Im Bereich der post-sozialistischen Gesellschaften sind in den letzten Jahren umfangreiche Forschungsprojekte durchgeführt worden, deren Schwerpunkte auf politischen Entscheidungsprozessen, Wirtschaftskultur und der EU-Osterweiterung lagen. Eine der Hauptaufgaben der Forschungsstelle ist die Information der interessierten Öffentlichkeit. Dazu gehören unter anderem regelmäßige E-Mail-Informationsdienste mit fast 15.000 Abonnenten in Politik, Wirtschaft und den Medien.

Mit ihrer in Deutschland einzigartigen Sammlung von Publikationen zu Osteuropa ist die Forschungsstelle eine Anlaufstelle sowohl für Wissenschaftler als auch für die interessierte Öffentlichkeit. In der Bibliothek sind derzeit neben anderen breit angelegten Beständen allein aus Polen ca. 300 laufende Periodika zugänglich. Die Bestände werden in Datenbanken systematisch erfasst. (www.forschungsstelle.uni-bremen.de)

Die Meinungen, die in den Polen-Analysen geäußert werden, geben ausschließlich die Auffassung der Autoren wieder. Abdruck und sonstige publizistische Nutzung sind nach Rücksprache mit der Redaktion gestattet. Redaktion: Prof. Dr. Dieter Bingen (Darmstadt), Silke Plate, M.A. (Bremen) Technische Gestaltung: Matthias Neumann

Polen-Analysen-Layout: Cengiz Kibaroglu, Matthias Neumann

ISSN 1863-9712 @ 2009 by Deutsches Polen-Institut Darmstadt und Forschungsstelle Osteuropa, Bremen

Kontakt: Dr. Andrzej Kaluza, Presse- und Öffentlichkeitsarbeit, Deutsches Polen-Institut, Mathildenhöhweg 2,

D-64287 Darmstadt, Tel.: 06151/4985-13, Fax: 06151/4985-10, E-Mail: polen-analysen@dpi-da.de, Internet: www.laender-analysen.de/polen 